\title{
Diabetes mellitus and male sexual function: a controlled study
}

\author{
R.C.Schiavi, B.B.Stimmel, J. Mandeli, E.J. Rayfield \\ Department of Psychiatry, Biomathematical Sciences and Medicine, Mount Sinai School of Medicine, New York City, New York, USA
}

\begin{abstract}
Summary. There is an extensive clinical literature on the erectile disorders of diabetic men but a paucity of controlled studies that have taken into account the effects of age, concurrent illnesses and medication on sexual function. This investigation was carried out on 40 diabetic men free from other illness or drugs that could affect sexual capacity and 40 age-matched healthy control subjects. Each subject and his female partner underwent semistructured interviews and the men had comprehensive medical evaluations and polygraphic assessment of sleep and nocturnal penile tumescence in the sleep laboratory during three nights. In comparison to control subjects, diabetic patients reported significant decreases in sexual desire, subjective arousal, erectile capacity, coital frequency and sexual satisfaction. The diabetic group also had significant decrements in duration of rapid eye movement sleep and in frequency, duration and degree of nocturnal penile tumescent episodes. There were no dif-
\end{abstract}

ferences between Type 1 (insulin-dependent) and Type 2 (non-insulin dependent) diabetic patients in prevalence of sexual problems or in nocturnal tumescent measures. Significant relations were observed between lack of metabolic control, diabetic complications and impaired nocturnal tumescence. Sexually non-dysfunctional diabetic men had significant nocturnal penile tumescence abnormalities. Diabetic men without coital failures may have a subclinical impairment in erectile function which, although of not significant magnitude to interfere with penetration, is reflected in nocturnal penile tumescent measures. This result raises a note of caution in the interpretation of the nocturnal penile tumescence test for the differential diagnosis of diabetic erectile impotence.

Key words: Diabetic impotence, nocturnal penile tumescence, sexual function, sexual disorders, sleep.
The association between diabetes mellitus and erectile impotence is well known but information about prevalence and the nature of this relationship remains unclear. Interpretation of prevalence estimates of diabetic erectile disorders ranging from 27 to $71 \%$ [1--3] is clouded by the lack of age-matched normal control subjects studied under similar conditions and by the confounding effects of concurrent medical illnesses and medications known to impair sexual activity. Understanding of the impact of diabetes on male sexual function is also limited by incomplete information on the relationship between diabetic characteristics and sexual variables, the narrow range of questions concerning sexual behaviour, lack of validation of the subject's report by the sexual partner, and absence of objective measures of erectile capacity.

In recent years, nocturnal penile tumescence (NPT) monitoring has been increasingly employed as a physiological test of organic impotence [4]. Diabetic patients with erectile disorders have shown significantly less NPT during rapid eye movement (REM) sleep than psycho- genically impotent and normal control individuals $[5,6]$. In a study from our laboratories [7], Type 1 (insulin-dependent) diabetic men free from erectile difficulties, had diminished penile circumference changes during sleep erections similar in degree to impotent diabetic patients. In addition, diabetic men with and without erectile difficulties spent less time in REM sleep and had longer latencies to REM onset. These observations have implications concerning the physiological processes that mediate sexual function in male diabetic patients, as well as practical consequences concerning the validity of the NPT method for differential diagnosis of organic impotence.

There are no controlled studies that have included psychosexual, sleep and NPT measures in a clinically welldefined diabetic group of men who are otherwise healthy and are not selected because of sexual problems. The present investigation was carried out on diabetic volunteers, living in stable sexual relationships, who were carefully screened to exclude the deleterious effects of other illnesses and drugs on sexual behaviour. The aims of the 
study were: 1) to compare a wide range of psychosexual variables between diabetic patients and age-matched healthy control subjects; 2) to compare sleep recordings and NPT parameters between the diabetic and control groups; 3 ) to assess the significance of diabetic type, metabolic control and diabetic complications on sexual function and NPT activity; and 4) to evaluate systematically NPT measures in diabetic men divided according to welldefined criteria of erectile impotence.

\section{Subjects and methods}

The subjects of this investigation included 40 diabetic men and 40 age-matched healthy volunteers. The inclusion criteria were 1) age less than 65 years, 2) married or living in a stable relationship with a female sexual partner for at least one year and 3) English as the primary language. The exclusion criteria were 1) concurrent illnesses other than diabetes or surgical interventions likely to impair sexual function, 2) drug use other than anti-diabetic medication, 3 ) history of alcoholism or other substance of abuse, 4) presence of major psychopathology and 5) obesity (more than $20 \%$ above ideal body weight as defined by the Metropolitan Life Insurance Tables). The healthy comparison group was selected according to the same criteria as the diabetic patients. Subjects were included in both groups regardless of the adequacy of their sexual functioning.

During a 4-year period, beginning in August 1987, 260 diabetic patients were referred by the faculty of the Mount Sinai Diabetic Clinic or contacted us in response to media announcements about the study. Two hundred and twelve patients were excluded mainly because of concurrent illnesses, use of non-diabetic medications, absence of sexual partner or inability or unwillingness to adhere to the study requirements. Eight subjects were eliminated primarily due to imcomplete data. Forty diabetic patients met entry criteria and completed all phases of the investigation. They were age-matched with a group of normal control subjects who participated in a study on healthy aging and were assessed under similar conditions. Informed consent was obtained from all subjects prior to the study, which was approved by the Institutional Review Board of the Mount Sinai Medical Center and was performed in accordance with the principles of the Declaration of Helsinki.

\section{Procedure}

Each subject and female partner underwent extensive joint and separate semistructured evaluations to obtain psychosexual, medical and marital information and to diagnose sexual disorders. A separate structured interview was conducted on each man with the use of the Schedule for Affective Disorders and Schizophrenia-Lifetime (SADS-L) [8] to identify drug abuse and psychopathology that would preclude study participation. In addition, men completed several questionnaires including the Michigan Alcoholism Screening Test [9] and the Mini-Mental State Examination [10] to rule out organicity. In the following session a comprehensive medical evaluation was carried out. Specific attention was paid to past and current history of coronary heart disease, hypertension, hepatic or renal insufficiency, central nervous system pathology such as multiple sclerosis or epilepsy, endocrinopathies and any other abnormalities which could predispose the subject to organic impotence. Additional reasons for exclusion were surgical interventions or accidental traumas likely to have impaired the neuromuscular components of erection.

Following the medical evaluation and chart review a detailed form was completed which included age of onset of diabetes, duration of diabetes, course, glycaemic control (glycohaemoglobin and self-glucose monitoring records), diabetic treatment and complications. Clinical evidence of peripheral and autonomic neuropathy, retinopathy and nephropathy was assessed according to a scoring system reported by McCulloch et al. [2]. Medical tests included blood chemistries, haematology, glycated haemoglobin determinations and urinalysis. Diabetic subjects were admitted to the Clinical Research Center where blood was obtained for hormonal assessment and a glucagon test and C-peptide determination were carried out to help characterize diabetic type. The results of the hormonal evaluation will be reported elsewhere. All subjects were asked to keep a daily record of sexual activities for the duration of the study or at least 30 days.

Polygraphic assessment of sleep and NPT was conducted in the sleep laboratory during three nights according to a previously described protocol [11]. Each subject arrived about $1 \mathrm{~h}$ before his usual bedtime and remained in bed until his usual rising time, providing that a minimum of $6 \mathrm{~h}$ of recorded sleep was obtained. Electroencephalography (EEG), eye movements, muscle tone and penile tumescence were monitored continuously through the night. Penile tumescence was measured by two strain gauge loops: one placed around the base of the penis and the other behind the corona of the glans. Sleep was undisturbed during the first two nights. During the third night visual checks were carried out during tumescent episodes to ascertain degree of erection in relation to the recorded increase in penile circumference. The subject and the observer separately rated the degree of erection on a $1-10$ scale where $1=$ none, $5=$ partial but adequate for penetration with manual assistance and $10=$ rigid. In addition, the technician noted the angle of the erection from the horizontal plane and obtained a photographic record. On one of the mornings after a sleep-recording night, penile vascular assessments were carried out in the Vascular Laboratory of the Department of Surgery by means of ultrasonic Doppler measurement and mercury strain-gauge plethysmography [12].

Sleep records were scored and quantified according to standardized criteria [13]. Sleep staging and NPT were graphed every $30 \mathrm{~s}$. Degree of NPT was defined as increase in penile circumference over the flaccid state, measured in millimeters at the base. Maximum and partial episodes were defined as deviations from the baseline recording of $81 \%-100 \%$ and $25 \%-80 \%$, respectively of the greatest circumference estimated to be full by direct observation. Frequency was defined as the mean number of total or maximum tumescence episodes per night or during REM sleep. Duration was defined as the mean time per night spent in tumescence, mean duration of maximum tumescence and mean duration of simultaneous REM sleep and tumescence.

\section{Statistical analysis}

The psychosexual variables of diabetic and normal men were compared by the Wilcoxon rank sum test. Dichotomous data were analysed by the chi-square test or Fishers's exact test. The sleep and penile tumescence variables were compared by the $t$-test for independent groups, except when the distributions of measurements within the groups were markedly skewed or had high kurtosis, in which case the Wilcoxon rank sum test was used.

For the comparisons of sleep and NPT variables of the diabetic men, categorized according to the severity of the erectile disorders, and the healthy control volunteers, the analysis of variance was employed along with Tukey's multiple comparisons procedure having an experiment-wise type 1 error rate of 0.05 . For data that was far from normally distributed, multiple Wilcoxon rank sum tests were carried out, each with a Bonferroni correction to the alpha level so that the experiment-wise type 1 error rate is 0.05 .

\section{Results}

\section{Clinical characteristics}

The mean age of the diabetic and control groups were $52.6 \pm 7.2(\mathrm{SD})$ years and $52.8 \pm 5.6$ years with the same median age of 53 years. The majority of the diabetic pa- 
Table 1. Psychosexual variables of diabetic patients and healthy control men

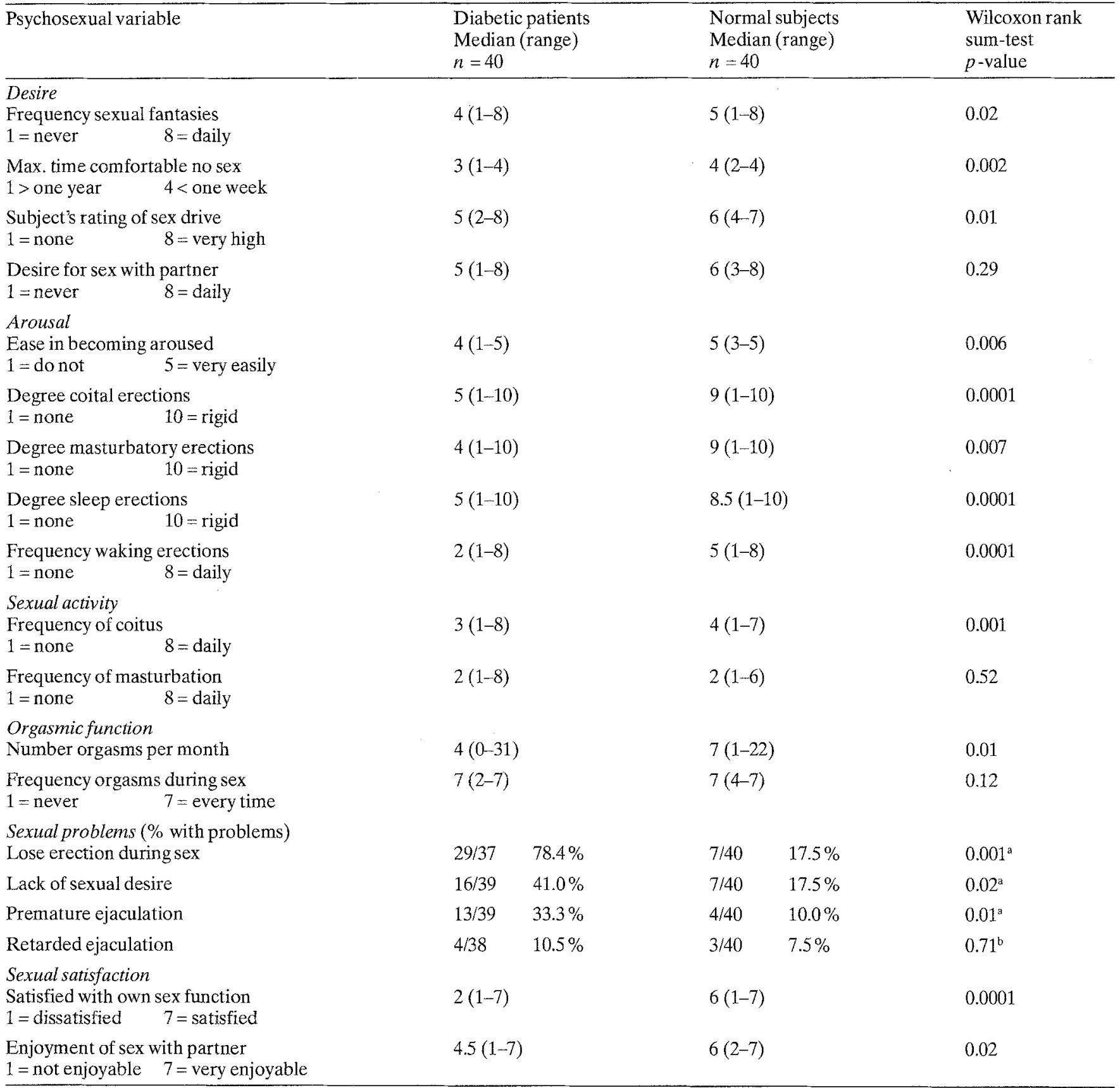

${ }^{a}$ Chi-square test; ${ }^{b}$ Fisher's exact test

tients were white $(77.5 \%) ; 7.5 \%$ were black and $10 \%$ were hispanic. Most of them were married (95\%) or living with their sexual partner; the mean duration of marriage was 22.1 years (range $1-42$ years) and the mean number of children was 2.5 (range 0-4). Ninety-two percent were high school graduates; $52.5 \%$ had a professional education; $25 \%$ had skilled or clerical jobs and $22.5 \%$ were retired. The racial composition of the control group was $97.5 \%$ white and $2.5 \%$ black. Other than a higher proportion of ethnic minorities represented in the diabetic group there were no significant demographic differences between the diabetic and control groups. The mean age at clinical onset of diabetes was 41.2 years (range 17-61) and the mean duration of the diabetic condition at the time of the study was 11.7 years (range 6 months-32 years). Based on clinical history and glucagon-stimulated C-peptide determinations nine subjects $(22.5 \%)$ were diagnosed as having Type 1 and 27 subjects $(67.5 \%)$ as having Type 2 (non-insulin-dependent) diabetes. Twenty-two patients $(55 \%)$ were receiving insulin, $13(32.5 \%)$ were on oral hypoglycaemic agents and $5(12.5 \%)$ were on diet regimens. Assessment of adequacy of metabolic control was based on historical information and glycated haemoglobin determinations. Glycated haemoglobin values above $8.0 \%$ were considered indicative of poor metabolic control. Diabetic control could be evaluated reliably in 37 patients; 21 subjects were judged to be in good or fair control and 16 in poor metabolic control. The proportion 
Table 2. Sleep and penile tumescence variables of diabetic patients and healthy control men

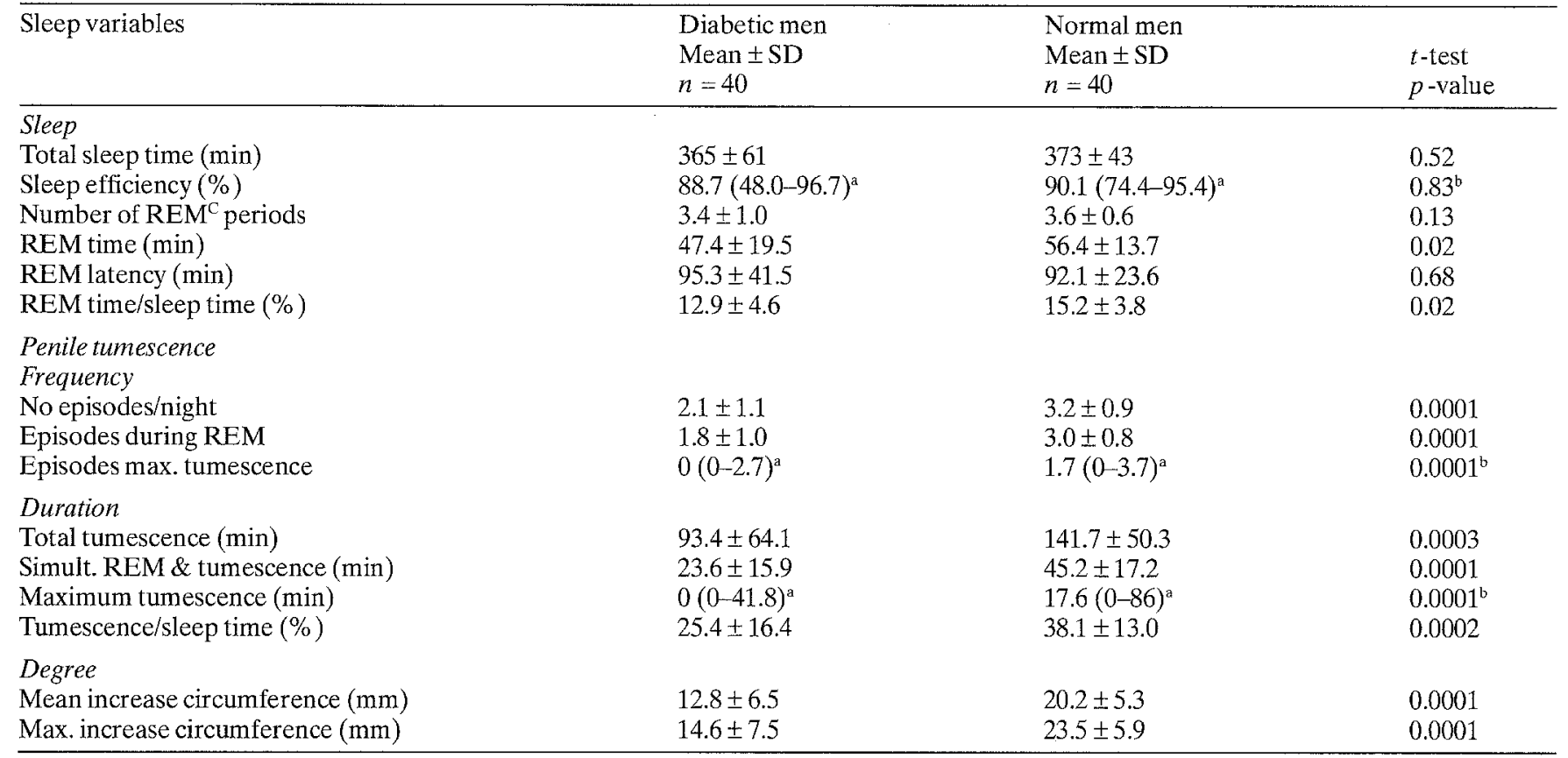

${ }^{a}$ Median (range); ${ }^{b}$ Wilcoxon rank sum-test; ${ }^{c}$ Rapid eye movement

of subjects with evidence of peripheral and autonomic neuropathy or retinopathy was 30 and $15.5 \%$, respectively. Twenty-eight subjects had no clinical evidence of diabetic complications.

Erectile dysfunction was diagnosed in 31 diabetic patients $(77.5 \%)$ and in 6 physically healthy control subjects $(15.0 \%)$ Nine diabetic subjects were categorized as having moderate and 22 as having severe impotence based on reported failure rates up to $50 \%$ and greater than $50 \%$ of coital attempts, respectively. Nine diabetic men who, although on occasion had noticed less than rigid erections but continued to have regular intercourse were classified as sexually non-dysfunctional. The mean age at the onset of erectile difficulties was 47.9 years (range 30-62) and the average duration of the problem was 5.1 years (range 6 months- 12 years). In eight patients the initial occurrence of erectile problems preceded the diagnosis of diabetes by 1 to 7 years. For the remaining men the onset of erectile problems occurred from 6 months to 23 years after diabetes was diagnosed.

Characteristically, most but not all, the diabetic patients reported the erectile difficulties as persistent, pervasive and as having developed progressively over time. Four sexually dysfunctional diabetic patients indicated adequate erections during sleep or masturbation even though their coital erections were severely impaired. All diabetic men retained orgasmic capacity but seven patients reported problems in the ejaculatory phase which included the sudden onset of premature or erratic ejaculation, decreased amount of ejaculate, "seepage" of semen at orgasm, or decreased orgasmic intensity. Finally, two subjects complained of decreased penile sensitivity and two others reported the occurrence of Peyronie's disease following the onset of diabetes.

\section{Behavioural variables}

The sexual dimensions assessed in the psychosexual interview of the diabetic and healthy control groups are summarized in Table 1. In comparison to control subjects, diabetic men showed significant mean decreases in sexual desire, subjective arousal, degree of coital, masturbatoryand sleep-related erections, frequency of coital activity and sexual satisfaction. In addition, a significantly higher proportion of diabetic men also reported problems with lack of sexual desire, erectile impotence and premature ejaculation.

\section{Sleep and nocturnal penile tumescent variables}

Sleep and NPT data averaged over three recording nights are summarized in Table 2 . There were no significant differences between the diabetic and healthy control groups in total sleep time, sleep efficiency, number of REM periods per night and REM latency defined as the length of time from onset of sleep (Stage 1) to the appearance of REM sleep. The diabetic group did have, however, significant decrements in duration of REM sleep and in the percentage of sleep time during REM. In addition they demonstrated consistent and highly significant decreases in frequency, duration and degree of nocturnal erectile episodes when compared to normal control subjects.

\section{Relationship between diabetic type, metabolic control, medical complications and sexual variables}

Comparison of psychosexual dimensions between Type 1 and Type 2 diabetic men and between patients in good 
Table 3. Sleep and tumescence variables in relation to metabolic control and complications

\begin{tabular}{|c|c|c|c|c|}
\hline \multirow[t]{3}{*}{ Sleep variables } & \multicolumn{4}{|c|}{ Diabetic patients (mean $\pm S D$ ) } \\
\hline & \multicolumn{2}{|c|}{ Metabolic control } & \multicolumn{2}{|c|}{ Complications } \\
\hline & $\begin{array}{l}\text { Good } \\
n=21\end{array}$ & $\begin{array}{l}\text { Poor } \\
n=16\end{array}$ & $\begin{array}{l}\text { Absent } \\
n=28\end{array}$ & $\begin{array}{l}\text { Present } \\
n=12\end{array}$ \\
\hline \multicolumn{5}{|l|}{ Sleep } \\
\hline Total sleep time (min) & $365 \pm 61$ & $365 \pm 62$ & $366 \pm 57$ & $362 \pm 72$ \\
\hline Sleep efficency $(\%)^{a}$ & $\begin{array}{l}85.9 \\
(48.1-96.3)\end{array}$ & $\begin{array}{l}90.2 \\
(47.9-96.7)\end{array}$ & $\begin{array}{l}89.8 \\
(48.1-96.7)\end{array}$ & $\begin{array}{l}87.7 \\
(47.9-96.3)\end{array}$ \\
\hline REM time ( $\min )$ & $46.9 \pm 18.0$ & $43.8 \pm 16.4$ & $47.8+20.1$ & $46.3 \pm 18.4$ \\
\hline REM latency (min) & $85.6 \pm 41.9$ & $108.1 \pm 40.9$ & $92.0 \pm 40.2$ & $102.9 \pm 45.2$ \\
\hline REM time/sleep time (\%) & $12.8 \pm 4.3$ & $12.0 \pm 3.9$ & $13.0 \pm 4.7$ & $12.9 \pm 4.6$ \\
\hline \multicolumn{5}{|l|}{ Penile tumescence } \\
\hline No episodes/night & $2.5 \pm 1.2$ & $1.6 \pm 0.9^{c}$ & $2.4 \pm 1.1$ & $1.4 \pm 1.0^{c}$ \\
\hline Simult. REM \& tumes. (min) & $25.5 \pm 15.1$ & $19.8 \pm 16.0$ & $26.3 \pm 16.6$ & $17.2 \pm 12.6$ \\
\hline Maximum tumescence (min) ${ }^{a}$ & $0(0-27.3)$ & $0(0-41.8)^{\mathrm{b}}$ & $0(0-41.8)$ & $0(0-7.7)$ \\
\hline Tumescence/sleep time (\%) & $33.1 \pm 16.3$ & $16.0 \pm 11.9$ & $29.4 \pm 15.0$ & $15.9 \pm 15.9^{\circ}$ \\
\hline \multicolumn{5}{|l|}{ Degree } \\
\hline Mean increase circumference (mm) & $14.5 \pm 5.9$ & $10.3 \pm 7.0^{\mathrm{b}}$ & $14.6 \pm 5.6$ & $8.6 \pm 6.7^{\mathrm{c}}$ \\
\hline Max. increase circumference (mm) & $16.8 \pm 6.9$ & $11.5 \pm 8.1^{\mathrm{b}}$ & $16.8 \pm 6.5$ & $9.7 \pm 7.8^{\circ}$ \\
\hline
\end{tabular}

${ }^{\mathrm{a}}$ Median (range); ${ }^{\mathrm{b}} p<0.05 ;{ }^{\mathrm{c}} p<0.01$; ${ }^{\mathrm{d}}$ Rapid eye movement

vs poor metabolic control demonstrated few significant behavioural differences. Type 1 diabetic patients were younger than the Type 2 group (47.4 vs 53.6 years, $p<0.03$ ). The degree of coital, masturbatory and nocturnal erections reported by Type 2 diabetic patients was significantly less than Type 1 diabetic men. The corresponding median (range) values in a 1-10 scale of erectile rigidity $(10=$ most rigid erection) were: coital $4(1-10)$ vs 8 $(5-10), p<0.03$; masturbatory $4(1-10)$ vs $7 \quad(3-10)$, $p<0.02$; sleep-related $4(1-10)$ vs $9.5(1-10), p<0.004$. There were no group differences, however, in the proportion of Type 1 and Type 2 patients reporting problems of sexual desire, erectile impotence, premature or retarded ejaculation.

A significantly higher proportion of patients in poor control compared to adequate metabolic status experienced an increased need for penile stimulation to reach orgasm. ( $73.3 \%$ vs $40.0 \%$, chi-square test $p<0.05$ ). Diabetic complications were associated with a greater difficulty in obtaining an erection during sexual activity $(p<0.01)$; and with decreased penile rigidity during intercourse $(p<0.05)$; a higher proportion of men with diabetic complications also stated that the increased time required to obtain an erection was a serious problem ( $91.7 \%$ vs $57.7 \%$; chi-square test $p<0.04)$. There was no relationship between adequacy of metabolic control and prevalence of diabetic complications. Twenty-nine percent of patients in good control and $31 \%$ of men in poor control suffered from clinical neuropathies or retinopathy. Diabetic patients categorized as having erectile impotence were similar to diabetic men free from sexual problems in diabetic type and adequacy of metabolic control but they differ markedly in frequency of complica- tions (dysfunctional diabetic patients $38.7 \%$ vs sexually normal diabetic patients $0 \%$ ).

There was extensive documentation of a relationship beween lack of metabolic control, diabetic complications and impaired nocturnal penile tumescence. As shown in Table 3, diabetic patients in poor control or with complications had significant decrements in frequency, duration and degree of sleep-related erections. It should be noted that there were marked differences in maximum tumescent episodes between subjects in good and poor metabolic control even though there were no group differences in the median number and duration of episodes per night. Forty-five percent of the subjects in good metabolic control exhibited episodes of maximum tumescence in comparison to only $7 \%$ in the poorly-controlled group. With the exception of a significant lower sleep efficiency in Type 2 compared to Type 1 diabetic men ( 87.0 vs $92.4 \%$, $p<0.05)$ there were no statistical differences in sleep and tumescent variables between the two diabetic types.

\section{Severity of erectile impotence and nocturnal penile tumescence}

The sleep and NPT variables of the diabetic men, categorized according to the severity of the erectile disorder, and the healthy control subjects without sexual problems were compared by analysis of variance and multiple comparison tests (Table 4). Diabetic men with severe potency problems were significantly older than diabetic subjects free from sexual difficulties but did not differ in age from the other two groups. With the exception of a significantly diminished duration of REM sleep in the severely dys- 
Table 4. Nocturnal penile tumescence variables in relation to adequacy of coital functioning

\begin{tabular}{|c|c|c|c|c|}
\hline \multirow[t]{2}{*}{ Penile tumescent variables } & \multicolumn{3}{|c|}{ Diabetic patients (mean \pm SD) } & \multirow{2}{*}{$\begin{array}{l}\text { Healthy non- } \\
\text { dysfunctional } \\
\text { subjects } \\
n=34\end{array}$} \\
\hline & $\begin{array}{l}\text { Dysfunctional } \\
\text { severely } \\
n=22\end{array}$ & $\begin{array}{l}\text { Dysfunctional } \\
\text { moderately } \\
n=9\end{array}$ & $\begin{array}{l}\text { Non- } \\
\text { dysfunctional } \\
n=9\end{array}$ & \\
\hline \multicolumn{5}{|l|}{ Frequency } \\
\hline No episodes/night & $1.7 \pm 1.2^{b}$ & $2.7 \pm 1.0$ & $2.5 \pm 0.9$ & $3.3 \pm 0.9$ \\
\hline Episodes during REM ${ }^{\mathrm{d}}$ & $1.5 \pm 1.1^{\mathrm{b}}$ & $2.4 \pm 0.9$ & $2.1 \pm 0.8^{\mathrm{b}}$ & $3.0 \pm 0.9$ \\
\hline Episodes max. tumes. ${ }^{\mathrm{a}}$ & $0(0-2.7)^{\mathrm{b}}$ & $0.33(0-2)^{b}$ & $0(0-1.7)^{\mathrm{b}}$ & $2.0(0-3.7)$ \\
\hline \multicolumn{5}{|l|}{ Duration } \\
\hline Total tumescence (min) & $65.9 \pm 53.7^{\mathrm{c}}$ & $134 \pm 56$ & $120 \pm 68$ & $147 \pm 51$ \\
\hline Simult. REM \& tumescence (min) & $19.1 \pm 16.0^{b}$ & $33.9 \pm 10.1$ & $24.2 \pm 17.8^{\mathrm{b}}$ & $46.6 \pm 18$ \\
\hline Maximum tumescence $(\min )^{\mathrm{a}}$ & $0(0-41.8)^{b}$ & $3.5(0-7.7)^{\mathrm{b}}$ & $0(0-27.3)^{\mathrm{b}}$ & $19.8(0-86.5)$ \\
\hline Tumescence/sleep time (\%) & $18.7 \pm 14.4^{\mathrm{c}}$ & $34.0 \pm 14.0$ & $32.9 \pm 17.2$ & $39.2 \pm 13.4$ \\
\hline \multicolumn{5}{|l|}{ Degree } \\
\hline Mean increase circumference $(\mathrm{mm})$ & $11.4 \pm 7.5^{b}$ & $15.2 \pm 4.1$ & $14.1 \pm 4.9^{\mathrm{b}}$ & $20.5 \pm 5.6$ \\
\hline Max. increase circumference (mm) & $12.8 \pm 8.6^{\mathrm{b}}$ & $17.4 \pm 5.3$ & $16.4 \pm 5.8^{\mathrm{b}}$ & $23.9 \pm 6.3$ \\
\hline
\end{tabular}

${ }^{\mathrm{a}}$ Median (range); ${ }^{\mathrm{b}}$ Significantly different from healthy non-dysfunctional men (multiple comparisons procedure, experimentwise error rate 0.05 ); ${ }^{c}$ Significantly different from all other groups (multiple comparisons procedure, experimentwise error rate 0.05 ); ${ }^{\mathrm{d}}$ Rapid eye movement

functional diabetic group, there were no statistical differencs among groups in any of the sleep variables. The frequency, duration and degree of NPT in severely impotent diabetic men were markedly and significantly less than in the healthy group. In addition, duration of total tumescence and the proportion of sleep time in tumescence in the severely impotent diabetic patients was significantly less than in the other two diabetic groups. The sexually non-dysfunctionl diabetic patients also showed significant decrements in frequency, duration and degree of NPT episodes in comparison to the healthy control subjects.

\section{Discussion}

There is an extensive clinical literature on the erectile disorders of diabetic men but a lack of controlled investigations that have taken into account the effect of age, concurrent illnesses and medication on sexual function [1-3, 14, 15]. The present study demonstrated that diabetic men, in comparison to age-matched healthy control subjects show extensive behavioural decrements in sexual desire, arousal, activity and satisfaction. The validity of these differences was confirmed by independent assessment of the sexual partners. In agreement with Fairburn and collaborators [16] the sexual problems were not limited to erectile impotence; they also included, decreased penile sensitivity and a range of ejaculatory/orgasmic disturbances. The abnormally diminished frequency, duration and degree of tumescent episodes during sleep paralleled the erectile difficulties reported by the diabetic patients. These results from patients unselected with regard to sexual functioning extend previous findings which have focused almost exclusively on impotent diabetic men.

There was little evidence that diabetes type is an important determinant of differences in sexual function. Type 2 diabetic men reported lower penile rigidity during sexual activity and early morning awakenings but it should be noted that they were older than the Type 1 diabetic patients. There were no differences between Type 1 and
Type 2 diabetic men in other psychosexual variables, in prevalence of sexual problems or in NPT parameters.

The effect of glycaemic control on the development of sexual problems is uncertain. Jensen observed that sexual dysfunction was unrelated to diabetic regulation [14] while in a prospective study McCulloch et al. [17] found that erectile impotence was associated with poor glycaemic control. In the present study lack of behavioural evidence of a relationship between metabolic control and sexual difficulties contrasts with the markedly decreased NPT activity measured in poorly-controlled diabetic patients. It seems reasonable to speculate that the widespread NPT decrements reflect a subclinical impairment that places the poorly-controlled diabetic patient at risk for eventual erectile difficulties.

The association of diabetic complications and sexual problems is in keeping with previous reports $[14,17,18]$. The markedly impaired NPT activity in diabetic patients with symptomatic neuropathy or retinopathy supports the notion that peripheral neurovascular pathology is involved in the pathogenesis of erectile impotence. Diabetic patients who met the criteria for erectile impotence in this study did have significantly diminished penile blood pressure (unpublished observation). The assessment of sleep architecture suggests, in addition, that abnormal central nervous processes may also contribute to diabetic erectile dysfunction. The observation of decreased sleep time devoted to REM confirm our previous findings leading to the hypothesis that a metabolic abnormality may disturb brain function and REM activity [7]. Although Nofzinger and Schmidt [19] did not observe a decrease in REM sleep, they found low REM density and a direct relationship between REM density and tumescence measures in impotent diabetic patients. There is growing evidence that a central autonomic dysregulation not involving peripheral autonomic pathology may be associated with deficient glucose metabolic control [20,21]. The possibility that a central autonomic dysfunction reflected in abnormal REM activity may also contribute to the erectile problems of diabetic men deserves further study [19]. 
Diabetic patients who had coital failure rates above $50 \%$ differed from matched non-dysfunctional healthy control subjects in all NPT measures; however, only duration of tumescence and the proportion of sleep time in tumescence differentiated the severely dysfunctional group from the diabetic men with less severe coital difficulties or with no erectile failures. The sexually non-dysfunctional diabetic men exhibited significant NPT abnormalities replicating our previous results [7] and the recent report by Nofzinger et al. [22].

We have speculated [7] that some diabetic men without coital difficulties may have a subclinical impairment in erectile function which, although of not sufficient magnitude to interfere with penetration, is reflected in NPT measures. Some of the diabetic subjects categorized a sexually non-dysfunctional in this study were concerned by a subtle decrease in penile rigidity even though they continued to have intercourse on a regular basis. NPT recordings may provide a sensitive indicator of physiologic changes in erectile capacity during the course of diabetes which had been previously ignored due to the exclusive focus on impotent patients. The observation that the moderately dysfunctional diabetic patients had less NPT decrements than the non-dysfunctional group suggests, however, that the relationship between coital failure rates and NPT measures is not a close one. Psychological and interpersonal factors, in addition to physiological capacity are likely to play an important role in the success or failure of coital experiences.

Conclusions from this study on a carefully selected group of patients should be generalized with caution. The results, unconfounded by the effect of unrelated medical disorders, clearly demonstrate that diabetes has a broad effect on male sexuality and that, physiologically, is associated with altered EEG activity and with NPT deficits which are also observed in patients who do not report coital failures. This finding has diagnostic relevance as NPT monitoring is being increasingly employed for the objective assessment of organic impotence. The diagnostic utility of the NPT method is based on the notion that in psychogenic impotence sleep erections are normal while in organic impotence nocturnal erections are impaired in correspondence to the patient's deficient waking erectile function. Present results suggest that impaired NPT may reflect subtle erectile deficiencies that are, nevertheless, compatible with regular coital activity. Before more information becomes available on coitally active diabetic men, care should be exercised in the interpretation of abnormal NPT findings for the differential diagnosis of diabetic erectile disorders.

Acknowledgements. The authors express their appreciation to Dr. D. E. Goldstein, Dr. S. Roman, Mr. D. White, Ms. D. Leben and Ms. S. Osborn. This research was carried out with the support of HSPHS research grant DK39106 and by a grant (5 M01 RR00071) for the Mount Sinai General Clinical Research Center from the National Center for Research Resources, National Institute of Health.

\section{References}

1. Schiavi RC, Hogan B (1979) Sexual problems in diabetes mellitus: psychological aspects. Diabetes Care 2:9-17
2. McCulloch DK, Campbell IW, Wu FC, Prescott RJ, Clarke BF (1980) The prevalence of diabetic impotence. Diabetologia 18: 279-283

3. Fairburn CG, McCulloch DK, Wu FC (1982) The effect of diabetes on male sexual function. Clin Endocrinol Metab 11: $749-767$

4. Schiavi RC (1988) Nocturnal penile tumescence in the evaluation of erectile disorders: a critical review. J Sex Marital Therapy 14:83-96

5. Karacan I, Salis PJ, Ware JC et al. (1978) Nocturnal penile tumescence and diagnosis in diabetic impotence. Am J Psychiatry 135: 191-197

6. Schiavi RC, Fisher C (1982) Assessment of diabetic impotence. Measurement of nocturnal erections. Clin Endocrinol Metab 11: 769-784

7. Schiavi RC, Fisher C, Quadland M, Glover A (1985) Nocturnal penile tumescent evaluation of erectile function in insulin-dependent diabetic men. Diabetologia 28: $90-94$

8. Spitzer RL, Endicott S (1975) Schedule for affective disorders and schizophrenia-lifetime version. New York State Psychiatric Institute Biometrics Research

9. Selzer ML (1971) The Michigan alcoholism screening test: the quest for a new diagnostic instrument. Am J Psychiatry 127: $1653-1658$

10. Folstein MF, Folstein SE, McHugh PR (1975) "Mini-Mental State": a practical method for grading the cognitive state of patients for the clinician. J Psychiatr Res 12: 189-198

11. Fisher C, Schiavi RC, Edwards A, Davis DM, Reitman M, Fine J (1979) Evaluation of nocturnal penile tumescence in the differential diagnosis of sexual impotence. Arch Gen Psychiatry 36: $431-437$

12. Schiavi RC (1992) Laboratory methods for evaluating erectile dysfunction in erectile failure: assessment and treatment. In: Leiblum SR, Rosen RC (eds). Guilford Press, New York, pp 141170

13. Rechteschaffen A, Kales A (1968) A manual of standardized terminology, techniques and scoring system for sleep stages. U.S. Government Printing Office, Washington DC

14. Jensen SB (1981) Diabetic sexual dysfunction: a comparative study of 160 insulin treated diabetic men and women and an agematched control group. Arch Sex Behav 10: 493-504

15. Fairburn CG (1981) The sexual problems of diabetic men. Br J Hosp Med 25: 486-491

16. Fairburn CG, Wu FC, McCulloch DK et al. (1982) The clinical features of diabetic impotence: a preliminary study. Br J Psychiatry 140: 447-452

17. McCulloch DK, Young RJ, Prescott RJ, Campbell IW, Clarke BF (1984) The natural history of impotence in diabetic men. Diabetologia 26: $437-440$

18. Clarke BF, Ewing DJ, Campbell IW (1979) Diabetic autonomic neuropathy. Diabetologia 17:195-212

19. Nofzinger EA, Schmidt HS (1990) An exploration of central dysregulation of erectile function as a contributing cause of diabetic impotence. J Nerv Ment Disease 178: 90-95

20. Vinik A, Mitchell B (1988) Clinical aspects of diabetic neuropathies. Diabetes Metab Rev 4: 223-253

21. Cryer PE (1992) Iatrogenic hypoglycemia as a cause of hypoglycemia in associated autonomic failure in IDDM. A vicious cycle. Diabetes 41: 255-260

22. Nofzinger EA, Reynolds CF, Jennings JR et al. (1992) Results of nocturnal penile tumescence studies are abnormal in sexually functional diabetic men. Arch Intern Med 152: 114-118

Received: 18 December 1992

and in revised form: 19 March 1993

Dr. R. C. Schiavi

Mount Sinai Medical Center

19 East 98 th Street, Box 1084

New York, NY 10029

USA 